\title{
Entre justice et psychiatrie: l'homosexualité dans le projet de Code pénal suisse (1918)
}

Thierry Delessert

\section{Summary}

In 1942 the Swiss penal code depenalises homosexual acts between agreeing adults under some conditions. The genesis of the penal article shows that it was constructed before the First World War and bears marks of the forensic theories of the turn of the century. Both by direct contacts and the authority of its eminent figures, Swiss psychiatry exerts an unquestionable influence on the depenalisation. The conceptualisation of homosexuality is also strongly influenced by the German psychiatric theories and discussed in reference to Germanic law. By the penal article, the Swiss lawyers and psychiatrists link the homosexual question with the determination of the irresponsibility of criminal mental patients and degeneracy.

Keywords: homosexuality; forensic psychiatry; Swiss and German psychiatrists; Swiss penal code; Auguste Forel

\section{Résumé}

En 1942, le Code pénal suisse dépénalise les actes homosexuels entre adultes consentant-es sous certaines conditions. La genèse de l'article pénal montre qu'il est construit avant la Première Guerre mondiale et porte la marque des théories psychiatriques du tournant des $\mathrm{XIX}^{\mathrm{e}}$ et $\mathrm{XX}^{\mathrm{e}}$ siècles. Par des contacts directs et par l'influence de ses figures de proue, la psychiatrie suisse exerce

\footnotetext{
* Cet article est issu de mon mémoire de maîtrise ès sciences politiques, et s'insère dans le cadre d'une recherche pour une thèse de doctorat dirigée par les professeurs Jean Batou et Vincent Barras à l'Université de Lausanne. Mes remerciements vont à V. Barras pour ses précieux conseils au cours de la rédaction de cet article.
}

Thierry Delessert, Institut d'histoire économique et sociale (IHES), Université de Lausanne, BFSH 2, CH-1015 Lausanne (thierry.delessert@unil.ch). 
un effet certain sur la dépénalisation. La conceptualisation de l'homosexualité est également fortement influencée par les théories psychiatriques allemandes et le droit germanique. Par l'article pénal, les juristes et les psychiatres suisses lient la question homosexuelle avec la détermination de l'irresponsabilité des malades mentaux criminels et la dégénérescence.

Le 23 juillet 1918, le Conseil fédéral présente au Parlement le projet de Code pénal suisse (CPS). Ce code, après délibérations et amendements des Chambres fédérales, est accepté en votation populaire le 3 juillet 1938. L'implémentation du CPS en 1942 achève l'unification du Droit suisse, débutée en 1888 par le professeur de droit pénal bernois Carl Stooss (1849-1934). Au cours des trente années de préparation du projet de CPS, la Société des médecins-aliénistes suisses (SMAS) a apporté une expertise pour toutes les dispositions pénales touchant les malades mentaux, et sur la détermination de leur dangerosité. Concernant l'homosexualité, une commission spéciale mandatée par la SMAS forge en 1913 un article pénal qui connaît peu de modifications lors de l'adoption populaire du CPS en décembre 1937. Cet article dépénalise les actes homosexuels entre adultes consentant-es dans la majorité des cantons suisses, et pénalise certains actes pour prévenir l'acquisition de l'homosexualité.

Les archives de la SMAS révèlent la formation d'un consensus sur la sémiologie de l'homosexualité et sur ses conséquences pénales entre 1910 et 1914. En résulte un véritable dispositif médico-légal qui montre les influences de plusieurs contextes et acteurs. D'une part, la catégorisation médicale de l'homosexualité en Suisse illustre l'influence de la psychiatrie de l'Asile du Burghölzli à Zurich et l'empreinte de l'une de ses figures éminentes: Auguste Forel. Ce psychiatre influence la dépénalisation des actes homosexuels, mais l'assortit d'une forte injonction d'invisibilisation, et l'inscrit dans des conceptions eugénistes particulières. D'autre part, le contexte pénal allemand pèse fortement sur la catégorisation de l'homosexualité en Suisse. En Allemagne, le droit germanique, répressif, favorise le développement de théories psychiatriques favorables à la dépénalisation, tout comme il provoque des scandales qui mènent à une tentative de renforcer les pénalités avant la Première Guerre mondiale. Enfin, le référentiel scientifique sur l'homosexualité est allemand, comme le révèlent a contrario les publications en français de PaulLouis Ladame. Ses traductions des principaux psychiatres en la matière, 
Krafft-Ebing, Moll et Hirschfeld, sont une référence reconnue dans le monde médical francophone de l'époque.

La lecture de la Chronique allemande de Ladame, tout comme la comparaison des éditions successives de La Question sexuelle de Forel, et l'analyse des écrits moraux et politiques de ce dernier sont cruciales pour objectiver l'influence des théories psychiatriques allemandes et du contexte pénal allemand sur la perception médico-légale de l'homosexualité en Suisse. Ces sources, croisées avec la Feuille Fédérale et des articles parus dans la Revue pénale suisse notamment, permettent de voir que la médicalisation de l'homosexualité est, à l'origine, un moyen de lutte contre un droit germanique réprimant les actes sexuels entre hommes, mais aussi le vecteur d'un nouvel ordre sexuel et moral placé sous le signe de la dégénérescence.

Une précision est nécessaire sur l'emploi du terme «homosexualité» dans cet article. Comme nous le verrons, il est relativement peu employé par les psychiatres du début du $\mathrm{XX}^{\mathrm{e}}$ siècle qui lui préfèrent les termes «inversion sexuelle» et «uranisme». En accord avec les auteurs contemporains sur l'homosexualité, son emploi en dehors des citations des auteurs de l'époque désigne le comportement sexuel en général, et concerne tant les hommes que les femmes.

\section{La catégorisation pénale de l'homosexualité en Suisse}

Le projet de CPS de 1918 punit la «débauche contre nature» par l'article 169:

1. La personne majeure qui aura commis un acte contraire à la pudeur sur une personne mineure du même sexe âgée de plus de seize ans, sera punie de l'emprisonnement pour un mois au moins.

2. Celui qui aura abusé de l'état de dénûment où se trouve une personne du même sexe que lui, ou de l'autorité que lui donne sur elle sa fonction, sa qualité d'employeur ou une relation analogue, pour lui faire subir ou commettre un acte contraire à la pudeur,

Celui qui fera métier de commettre des actes contraires à la pudeur avec des personnes du même sexe,

Sera puni de l'emprisonnement pour un mois au moins ${ }^{1}$.

Cet article pénal deviendra, sous une forme épurée introduisant la notion de séduction dans le premier alinéa, l'article 194 du CPS de 1942, abrogé en

1 FF IV 1918, 168. 
$1992^{2}$. Il introduit la dépénalisation des actes homosexuels entre adultes consentant-es des deux sexes dans la majorité des cantons suisses, et sera vivement débattu entre 1920 à 1931 dans les commissions et dans les séances plénières des deux chambres fédérales, au point que le rapporteur de la Gazette de Lausanne qualifie en mars 1929 les débats du Conseil national de «Cour du Roi Pétaud» ${ }^{3}$. Lors des débats parlementaires, l'opposition entre les députés des cantons latins, permissifs, et germaniques, répressifs, et entre les catholiques conservateurs et les socialistes alémaniques, sera marquée sur cette question, mais déborde de cette contribution ${ }^{4}$.

En se concentrant sur la période de la construction du projet de CPS jusqu'à la fin de la Première Guerre mondiale, l'article 169 illustre le caractère novateur d'un droit «éclairé» par les nouvelles sciences, et plus particulièrement la psychiatrie légale. Ainsi, le Message du Conseil fédéral à l'appui du projet de CPS précise en 1918 que la catégorisation de l'homosexualité dans le CPS est directement influencée par les «aliénistes» suisses, et s'inscrit dans le courant de l'anthropologie criminelle et de l'école positive italienne ${ }^{5}$. Ce courant de positivisation et de psychiatrisation de la criminalité permet aux psychiatres d'introduire les notions d'irresponsabilité et de responsabilité restreinte, ainsi que des mesures d'expertise et de sûreté spécifiques pour les malades mentaux, dans le CPS. En se distinguant du corps médical, les aliénistes reçoivent la reconnaissance de leur exclusivité dans la connaissance scientifique et dans le traitement des maladies mentales ${ }^{6}$.

La médicalisation de l'homosexualité a pour principal effet d'introduire sa dépénalisation en Suisse, et permet de dépasser les clivages entre les cantons. En effet, dans une étude comparative des dispositions cantonales sur les mœurs, le juge genevois Ernest Picot (1853-1921) relève en 1889 que «la divergence qui existe entre les législations allemandes et romandes n'est nulle part aussi marquée que lorsqu'il s'agit de la répression de l'inceste et

2 Suite à la modification des infractions contre les mœurs en 1992, l'homosexualité est totalement dépénalisée en Suisse. La majorité pour toute orientation sexuelle est fixée à 16 ans, et l'abus de détresse est étendu aux deux sexes par le nouvel art. 193. La prostitution masculine est assimilée à la prostitution féminine et soumise aux mêmes dispositions concernant «l'encouragement de la prostitution» selon l'art. 195 CPS 1992. Sur les motifs, cf. FF II 1985, 1024 et $1103-1104$.

3 Gazette de Lausanne, 15 mars 1929,1.

4 Sur les débats des années 1920 sur l'ensemble des articles pénaux concernant la sexualité, cf. Gerodetti 2005.

5 FF IV 1918, 6 et 48.

6 Sur les questions de responsabilité et de psychiatrisation de la criminalité, cf. Bomio 1990; Gasser 2000a; Gasser/Barras 2000. Il manque un ouvrage général sur l'histoire de l'aliénisme en Suisse. Pour un dossier thématique sur l'histoire de la psychiatrie en Suisse, cf. Traverse 2003/1. Pour une bibliographie des études et des sources couvrant le champ de la psychiatrie suisse, cf. Gasser 2000b. Pour une illustration de la conception des institutions psychiatriques suisses, cf. Fussinger/Tevaearai 1998. 
des actes contre nature» ${ }^{7}$. Avant la Première Guerre mondiale, seuls les cantons de Genève, de Vaud, du Valais, de Fribourg, du Tessin et de Zurich ${ }^{8}$ ne criminalisent pas l'homosexualité entre adultes consentant-es. Ils seront rejoints par Bâle-Ville en 1919, sous l'influence du projet de CPS. La période d'avant-guerre en Suisse fait surtout apparaître un clivage entre le code napoléonien ${ }^{9}$ et le code germanique ${ }^{10}$, à l'exemple du canton de Neuchâtel qui réintroduit une peine pour homosexualité sous l'influence des débats allemands, en $1891^{11}$. Paradoxalement, le clivage confessionnel entre les cantons protestants et catholiques ne permet pas de marquer une ligne entre la tolérance ou la répression de l'homosexualité, alors que cette opposition sera plus marquée au cours des débats parlementaires des années 20.

Toutefois, il serait erroné d'inscrire totalement la dépénalisation de l'homosexualité en Suisse dans une extension du droit français, car la construction du fait homosexuel, au tournant des $\mathrm{XIX}^{\mathrm{e}}$ et $\mathrm{XX}^{\mathrm{e}}$ siècles, se trouve sous la forte influence des théories médico-légales allemandes et du contexte pénal en vigueur dans ce pays. Comme nous le verrons plus loin, certains psychiatres allemands obtiennent l'acquittement d'homosexuels lors de procès, en invoquant l'irresponsabilité de leurs actes pour cause de maladie mentale. La médicalisation de l'homosexualité s'inscrit donc dans une perspective de libération des «vrais» homosexuels et de lutte contre un droit germanique répressif.

\section{L'article pénal suisse: un dispositif médico-légal}

Le processus de catégorisation de l'homosexualité est double au début $\mathrm{du} \mathrm{XX}^{\mathrm{e}}$ siècle: la sémiologie psychiatrique et les conséquences pénales se construisent parallèlement. D'une manière générale, la psychiatrie légale

7 Picot $1889,65$.

8 Le code pénal zurichois punit la débauche contre nature, mais l'article n'est appliqué que pour la prostitution (Hafter 1929, 44).

9 Le code napoléonien de 1810, reprenant le code pénal adopté par l'Assemblée constituante révolutionnaire en 1791, introduit la dépénalisation de l'homosexualité. Presque tous les Etats envahis par Bonaparte, qu'ils soient catholiques ou protestants - l'Italie du Nord, la Belgique, les Pays-Bas, des Länder allemands, dont la Bavière, le Wurtemberg et le Hanovre, et les cantons suisses romands -, ont adopté cette dépénalisation (Leroy-Forgeot 1997).

$10 \mathrm{La}$ réclusion pour sodomie est introduite dans le code pénal prussien en 1794, et remplace la peine de bûcher introduite par Charles-Quint en 1532 dans le droit germanique. Il s'agit du premier code européen à modérer la peine contre l'homosexualité. L'ensemble des pays et cantons germaniques introduisent cette disposition au début du XIX ${ }^{\mathrm{e}}$ siècle (LeroyForgeot 1997).

11 Neuchâtel avait aboli la réclusion de l'homosexualité lors de la proclamation de la République en 1855. Dans le code de 1891, la poursuite ne peut être faite que sur plainte, et des mesures de médiation sont prévues (Ladame 1914, 293sq.). 
alors en cours de constitution définit à la fois son domaine, son expertise, et la norme légale sur une variété de préoccupations médicales et sociales, allant de la définition pénale de la responsabilité, de la place de l'expertpsychiatre dans les procès, à la lutte contre l'alcoolisme ou pour la protection de la jeunesse. La question de l'homosexualité illustre la construction d'un modèle préventif dans le CPS, basé sur les conséquences des notions d'irresponsabilité et de responsabilité.

Par la catégorisation d'actes qualifiés «contre nature», l'article pénal opère une distinction fondamentale entre une homosexualité innée - irresponsabilisée et non pénalisée - et une homosexualité acquise - responsabilisée et pénalisée. La prévention de l'acquisition de l'homosexualité est obtenue par l'élévation de l'âge de la majorité sexuelle homosexuelle, par l'introduction d'un abus de dépendance spécifiquement homosexuel, et par l'interdiction de la prostitution homosexuelle. Enfin, fait novateur pour l'époque ${ }^{12}$, l'article pénal s'applique tant pour les hommes que pour les femmes.

Les archives de la Société Suisse de Psychiatrie révèlent un contact entre les aliénistes et les juristes pour forger l'article pénal. Une commission spéciale formée par les psychiatres Eugen Bleuler (1857-1939), directeur de l'Asile du Burghölzli à Zurich, Ludwig Frank (1863-1935), ancien directeur de l'Asile de Münsterlingen en Thurgovie installé à Zurich, et Hans Maier (1882-1945), second médecin à l'Asile du Burghölzli, est nommée en juin 1911 par la Société des médecins aliénistes suisses. Ils sont chargés par leurs pairs de préparer des articles pénaux conformes aux résolutions adoptées: le combat contre l'homosexualité avec des mineurs, la lutte contre l'homosexualité acquise, considérée comme un défaut moral, et la non-pénalisation des actes homosexuels entre adultes consentant-es ${ }^{13}$. Cette commission rencontre les experts nommés par le Conseil fédéral pour l'élaboration finale du CPS, le 18 avril 1913. Au terme de la discussion, les alinéas sur la protection de la jeunesse, sur l'abus de détresse et sur la prostitution sont retenus pour former l'article 169 du projet de $\mathrm{CPS}^{14}$.

12 Les lesbiennes ne sont pas punies dans les codes pénaux germaniques et anglo-saxons du début du XX $X^{\mathrm{e}}$ siècle (Tamagne 2000; Leroy-Forgeot 1997).

13 SSP 1914.

14 Ladame 1914,281. Il est à relever qu'une seconde délégation de psychiatres sera nommée en 1929 pour défendre l'article devant la commission de renvoi du Conseil national. Cf. note 28 dans cet article. 


\section{Les débats au sein de la Société des médecins aliénistes suisses (SMAS)}

Fondée en 1864, la SMAS, qui devient en 1919 la Société Suisse de Psychiatrie, se réunit deux fois par année dans les principaux asiles suisses. Les réunions permettent à chacun de ses membres d'exposer les résultats de sa pratique, et les protocoles rendent compte de la constitution d'un savoir médical sur les psychopathologies en général, tout comme ils donnent des échos concrets sur les priorités des directeurs d'asiles psychiatriques confrontés à un manque de moyens. D'un point de vue plus strictement médico-légal, l'ensemble des articles du projet de CPS ayant un lien avec les maladies mentales sont passés en revue avant la Première Guerre mondiale, et assortis de diverses recommandations à l'intention des experts nommés par le Conseil fédéral.

Les débats sur la sémiologie et sur la catégorisation pénale de l'homosexualité se tiennent au cours de trois réunions, entre 1910 et 1914. Lors de la $43^{\mathrm{e}}$ rencontre de la SMAS, en novembre 1910 à Berne ${ }^{15}$, deux propositions d'ajouts sont mises à l'ordre du jour: l'interdiction de la propagande ouverte de l'homosexualité et l'introduction d'une peine de prison ou de maison de correction sans limite de temps pour les actes commis par un majeur sur un mineur de plus de seize ans. L'interdiction de la propagande homosexuelle, une proposition d'Auguste Forel (1848-1931), ancien directeur de l'Asile du Burghölzli installé dans le canton de Vaud, est rejetée par la SMAS. Selon Fritz Ris (1867-1931), directeur de l'asile rural zurichois de la Rheinau, une telle mesure risque «de limiter la littérature psychiatrique sur ce sujet» ${ }^{16}$. En revanche, l'extension de la peine est adoptée, mais assortie de la recommandation d'inviter les autres associations médicales suisses afin d'en discuter la portée. En effet, Maier, qui sera nommé à la commission de 1911, argumente qu'il ne faut pas introduire des «dispositions médicales fausses» dans le $\mathrm{CPS}^{17}$. On peut émettre l'hypothèse que la discussion entre les associations médicales remet en cause une telle définition de la peine, puisque la disposition ne se retrouve pas dans le CPS.

La 44 ${ }^{\mathrm{e}}$ réunion de la SMAS de juin 1911 à l'Asile de Cery près de Lausanne $^{18}$ est cruciale pour la catégorisation pénale de l'homosexualité en Suisse. Avant la nomination de la commission spéciale formée par Bleuler, Frank et Maier, la question de l'homosexualité est développée en allemand par Forel. Dans un long exposé, il défend sa position en faveur de la dépénalisation des actes homosexuels entre adultes consentant-es, et propose de ren-

15 SSP 1910/2.

16 SSP $1910 / 2,6$.

17 SSP 1910/2, 6, souligné dans le protocole.

18 SSP 1911/1. 
forcer la protection de la jeunesse. Pour ce psychiatre, la question de l'homosexualité est mal abordée: «Les homosexuels sont des malades, et le rôle du droit est de protéger les personnes à moitié saines de cette tendance», soit les jeunes et les «bisexuel-les», selon notre terminologie actuelle ${ }^{19}$. Les protocoles révèlent un accord des aliénistes présents avec les conceptions de Forel; une convergence qui sera examinée plus loin.

En juin 1914, au cours de la 50é réunion de la SMAS à l'asile thurgovien de Münsterlingen ${ }^{20}$, le psychiatre et neurologue genevois Paul-Louis Ladame (1842-1919) expose ses «remarques psychiatriques $»^{21}$ sur l'article pénal forgé par ses confrères. Ladame approuve la non-poursuite des homosexuel-les adultes ainsi que l'égalité prévue entre l'homosexualité féminine et masculine. Cependant, il estime que l'alinéa sur l'abus de détresse ne protège pas suffisamment les adultes infirmes, et que l'interdiction de la prostitution homosexuelle ne préserve pas la jeunesse car elle serait pratiquée par «les mineurs de plus de 16 ans [...] qui forment la grande majorité des pédérastes passifs faisant métier de leur vice et qui n'appartiennent que rarement aux vrais homosexuels» ${ }^{22}$. Ladame propose de supprimer toute poursuite contre l'homosexualité, et d'étendre les trois alinéas aux deux sexes. Au terme d'un bref débat, la proposition est rejetée par l'adoption, à l'unanimité des membres présents, d'une déclaration rappelant les résolutions prises en 1911 et se ralliant au travail effectué par la délégation Bleuler/Frank/Maier ${ }^{23}$.

Les comptes-rendus des débats sur l'homosexualité au sein de la SMAS sont relativement brefs en comparaison d'autres thèmes comme la schizophrénie ou la notion de responsabilité, voire avec la question de l'épilepsie comme pathologie psychiatrique au cours des mêmes années avant-guerre. Ils démontrent une convergence nette des psychiatres suisses sur la distinction entre l'homosexualité innée et acquise, et sur le dispositif médico-légal découlant de cette conceptualisation. Ainsi, la divergence de Ladame sur l'article pénal apparaît plus de forme que de fond, ses buts explicites étant la prévention des chantages et des «scandales» homosexuels similaires à ceux touchant l'Allemagne, en raison de l'existence d'une pénalité spécifique, et la volonté d'éviter des conflits d'interprétation ultérieurs ${ }^{24}$. Il marque également son plein accord avec les progrès scientifiques qui permettent de mettre en évidence les «racines biologiques de l'homosexualité» et de «soustraire sa compréhension pénale du droit canonique» ${ }^{25}$.

19 SSP 1911/1, 31.

20 SSP 1914.

21 Ladame 1914.

22 Ladame 1914, 290.

23 SSP 1914, 919.

24 Ladame 1914, 290.

25 Ladame 1914, 284sq. 


\section{Une science du Burghölzli}

L'absence d'un conflit profond entre les psychiatries suisses romands et alémaniques sur la question de l'homosexualité est notable. D'une manière générale, André Repond (1886-1973), directeur de l'Asile de Malévoz (Valais), relève en 1936 que les réunions tenues par la SMAS avant la Première Guerre mondiale ont produit «une entente sur la classification des maladies mentales [avec] pour résultat de créer une certaine unité dans la manière de considérer quelques problèmes psychiatriques fondamentaux». D'autre part,l'unité théorique des premiers temps de la psychiatrie suisse est marquée «par une seule école: celle du Burghölzli à Zurich» ${ }^{26}$. Le consensus sur la catégorisation pénale de l'homosexualité et la composition exclusivement zurichoise de la délégation médicale illustrent cette empreinte. D'abord, l'ensemble des aliénistes identifiés ont fait leurs études à Zurich et en Allemagne à cause de l'absence d'une faculté de médecine en Suisse romande. Ensuite, la réunion tenue à l'Asile de Cery près de Lausanne en juin 1911 montre que le dispositif médico-légal est directement soutenu par deux psychiatres qui ont fait la renommée de l'Asile du Burghölzli, soit Bleuler et Forel.

Bleuler demeure une référence incontournable dans l'étude des psychopathologies, et de la psychose plus précisément. Argumentant surtout sur la notion de responsabilité restreinte, en comparaison du droit allemand, ses propos apparaissent laconiques sur la question de l'homosexualitée ${ }^{27}$. En comparaison, Forel, son prédécesseur ${ }^{28}$, influence profondément la conception médico-légale de l'homosexualité en Suisse. Depuis la parution de $L a$ Question sexuelle exposée à des adultes cultivés, en 1905 en allemand et en

26 Repond 1936, 5.

27 Par exemple, l'unique page consacrée à ce thème dans son Lehrbuch der Psychiatrie relève le caractère aléatoire de l'hérédité, et souligne une bonne connaissance en général de l'homosexualité au regard d'une population estimée à 1\% (Bleuler 1916, 421). Toutefois, Bleuler sera remercié pour son influence sur la dépénalisation de l'homosexualité en Suisse par le journal homosexuel Menschenrecht en 1942 (Trüeb/Miescher 1988, 191; Kennedy 1999). Ce fait ouvre une perspective pour saisir les divergences de vue entre Suisses romands et alémaniques au cours des années 1920 et 1930.

28 Il est intéressant de relever le réseau des psychiatres composant la commission mandatée par la SMAS en 1911: tant Bleuler que Frank ont été des assistants de Forel, et sont depuis des amis. Ils ont été tous les deux nommés directeurs d'asile sur sa recommandation, et se sont pleinement engagés dans sa lutte contre l'alcoolisme et les conduites déviantes (Forel 1941). Proche de Frank, disciple et successeur de Bleuler à la direction de l'Asile du Burghölzli, Maier gardera des contacts comme expert avec les différentes commissions du Conseil national au cours des années 20 (Hafter 1929). Il sera à nouveau nommé membre de la délégation de psychiatres, en 1929, avec Repond et Oscar Forel (1891-1982). Contrairement à ce qu'affirme Gerodetti, Auguste Forel n'est pas présent lors de la rencontre du 14 novembre 1929 avec les députés de la commission de renvoi du Conseil national (Gerodetti 2005, 77 et s.). 
1906 en français, il est considéré comme le spécialiste suisse des perversions sexuelles. Ses écrits (en français), comme ceux de Ladame, montrent que le débat médical sur l'homosexualité est dominé par les théories psychiatriques allemandes, et argumenté en référence au $\$ 175$ du code pénal allemand ${ }^{29}$. Par conséquent, avant de cerner les conceptions originales de Forel, un éclairage sur les contextes légaux, psychiatriques, et politiques allemands est nécessaire pour comprendre la perception de l'homosexualité au sein de la psychiatrie suisse du début du $\mathrm{XX}^{\mathrm{e}}$ siècle.

\section{Le contexte médico-légal allemand}

$\mathrm{Au}$ tournant des $\mathrm{XIX}^{\mathrm{e}}$ et $\mathrm{XX}^{\mathrm{e}}$ siècles, tout auteur érudit sur la question de l'homosexualité se réfère aux théories des psychiatres allemands, et à leurs conséquences sur les plans pénaux et sociaux. L'allemand est la langue scientifique sur l'homosexualité. Les termes homosexualité et homosexuel sont employés pour la première fois à Leipzig en 1869 dans un pamphlet publié par un juriste hongrois, Karolay Maria Kertbeny (1824-1882). L'homosexualité est décrite comme un comportement sexuel normal, égal sur un plan juridique à l'hétérosexualité, un autre terme également inventé par Kertbeny ${ }^{30}$. Le terme d'homosexualité est peu employé au début du $\mathrm{XX}^{\mathrm{e}}$ siècle, et ne va s'imposer dans le langage médical et populaire qu'au cours des années 30. Il est concurrencé dans le langage scientifique par la notion de «Conträre Sexualempfindung», traduite par sentiment sexuel contraire, sensation sexuelle inverse, ou instinct sexuel inverse, et inventée en 1870 par Carl Westphal (1833-1890), médecin-chef du Département des maladies mentales de la Charité royale du Hanovre, ainsi que par le terme d'uranisme apparu en 1864 sous la plume d'un assesseur du Royaume de Hanovre, Karl Heinrich Ulrichs (1825-1895).

En Allemagne, le regard juridique et médical sur l'homosexualité est fortement influencé par sa pénalisation. En effet, le code pénal prussien promulgué en 1851 comprend un article punissant par la prison la «débauche contre nature». Entre 1867 et 1872, cet article est étendu à l'ensemble du

29 De manière flagrante, les codes pénaux des cantons germaniques proches du code pénal allemand ne sont pas abordés par les médecins suisses, et ne suscitent aucune critique. D'autre part, les psychiatres identifiés proviennent de cantons qui ne pénalisent pas l'homosexualité, et paraissent socialisés à une forme de tolérance, comme l'exemplifie Ladame: «Les pays et les cantons suisses qui ne poursuivent pas les <actes contraires à la pudeur sur les personnes de même sexe> ne paraissent pas avoir à se plaindre de la propagation de la pédérastie, comme c'est le cas surtout dans les pays qui la punissent» (Ladame 1914, 293; souligné par l'auteur).

30 Kennedy 1997; Katz 2001. Pour une biographie de Kertbeny, cf. Feray/Herzer 1990. 
Reich pour devenir le $\$ 175$ qui restera en vigueur durant plus d'un siècle ${ }^{31}$. L'extension du code pénal prussien réintroduit la pénalisation de l'homosexualité dans les Länder qui avaient adopté le code napoléonien, et c'est dans ce contexte que Ulrichs, Kertbeny et Westphal publient leurs appels à la dépénalisation de l'homosexualité 32 . Le $\$ 175$ punit les actes sexuels entre hommes de 1 jour à 5 ans de prison et force le regard médical à se concentrer sur l'homosexualité masculine. D'ailleurs, les psychiatres de l'époque relèvent que ce sont surtout des hommes qui sont soumis à une expertise psychiatrique lors de procès. Les cas d'homosexualité féminine ne semblent connus qu'en cas de divorce, voire chez des prostituées ${ }^{33}$.

\section{Les théories psychiatriques allemandes}

Les médecins de l'époque s'accordent en attribuant la paternité de la catégorisation de l'homosexualité à trois psychiatres, Krafft-Ebing, Moll et Hirschfeld, et relèvent l'opposition de deux conceptions de l'homosexualité innée: dégénérée ou naturelle. La Chronique allemande de Ladame offre un excellent exposé des théories et controverses allemandes. Publiée entre 1893 et 1913 dans les Archives d'anthropologie criminelle et de médecine légale, elle montre en Ladame un commentateur francophone averti des avancées de l'anthropologie criminelle et de la psychiatrie allemande en général, et de l'homosexualité en particulier ${ }^{34}$. Par ses prises de position, Ladame indique clairement les effets des théories allemandes et des rivalités politiques allemandes sur le processus de catégorisation de l'homosexualité alors en cours en Suisse.

La Psychopathia Sexualis du professeur de psychiatrie viennois Richard von Krafft-Ebing (1840-1902) est l'ouvrage majeur sur les «déviances sexuelles», et sur leurs conséquences pénales. Dans sa première Chronique allemande, Ladame la signale dans ces termes:

31 Le $\$ 175$ est modifié en 1969 en RFA et ne punit plus que les actes commis par un majeur sur un mineur de 16 à 18 ans. Il est définitivement aboli suite à la réunification en 1994. Le $\S 175$ avait été, par contre, complètement aboli en RDA en 1964 (Leroy-Forgeot 1997).

32 Cardon 1993.

33 Praetorius 1912; Ladame 1913; Forel 1906.

34 En 1890, Julien Chevalier (1860-1943), l'un des précurseurs de la diffusion du modèle médical (allemand) de l'homosexualité en France, avec Jean Martin Charcot (1825-1893) et Valentin Magnan (1835-1916), signale Ladame comme un «observateur attentif des théories sur l'inversion sexuelle et ses nouveaux symptômes» (Chevalier 1890,326). D'autre part, Ladame est un des rares auteurs à utiliser couramment le terme homosexualité pour légitimer son approche scientifique. 
Les aberrations du sens génésique sont traitées avec de grands détails par le professeur von Krafft-Ebing dans un volume de la Psychopathia Sexualis qui compte chaque année une nouvelle édition, de plus en plus augmentée, tant le thème des perversions sexuelles est inépuisable. Le livre du professeur de Vienne a été vivement critiqué à Berlin. Il renferme un chapitre sur les délits et les crimes sexuels dans lequel il insiste avec beaucoup de raison sur la nécessité de soumettre à l'examen d'experts médicaux les délinquants de cette espèce. L'acte délictueux ne peut jamais, à lui seul, indiquer son origine pathologique [...]. Un examen clinico-anthropologique complet est seul capable de trancher la question d'immoralité ou de psychopathie..$^{35}$

Pour Krafft-Ebing, l'homosexualité est un stigmate fonctionnel de la dégénérescence. Sa classification place l'instinct sexuel contraire dans les névroses sexuelles, plus précisément, dans les paresthésies, ou perversions de l'instinct sexuel, qui comprennent également l'amour cruel et sanglant, l'anthropophagie, la flagellation active et passive, le penchant pour un objet inanimé, l'exhibition, la nécrophilie et la bestialité. Pour les invertis-nés, chez qui existe exclusivement le penchant pour le même sexe, et dont la dégénérescence peut être prouvée par l'expertise psychiatrique, Krafft-Ebing postule l'abandon des poursuites pénales en faveur de mesures médicales ${ }^{36}$.

Le neurologue et psychiatre berlinois Albert Moll (1862-1939) est la deuxième personnalité marquante dans la catégorisation de l'homosexualité. En 1891, sa première publication, Die Conträre Sexualempfindung («L'inversion sexuelle»), reprend le terme de Westphal, et est largement traduite par Ladame dès la Chronique allemande de 1893. Moll sépare l'homosexualité innée et acquise, mais considère cette dernière comme exceptionnelle. Pour lui, des prédispositions existent antérieurement au passage à l'acte dans des cas «d'homosexualité tardive»; une tendance refoulée, soit par la peur des conséquences pénales, soit par la moralité du sujet, ou encore par l'absence de possibilité de rencontres avec des personnes de même sexe. Cette considération introduit donc l'idée qu'une personne peut être un homosexuel «latent», sans avoir passé à l'acte, et élargit la catégorie des actes désignés homosexuels. Marquant son accord avec la théorie de la dégénérescence défendue par Krafft-Ebing, Moll préconise l'abstinence absolue pour traiter l'homosexualité:

Il ne faut pas chercher à transformer l'homosexualité en hétérosexualité, ne serait-ce déjà en raison du danger de la transmission héréditaire de cette dégénérescence. Le traitement par la suggestion hypnotique sera utile pour réprimer l'instinct sexuel perverti. ${ }^{37}$

En reprenant la conception de Westphal, Krafft-Ebing et Moll catégorisent d'emblée l'homosexualité comme une maladie mentale. En donnant une large audience aux termes d'inversion sexuelle et d'inverti dans le langage scientifique, ils lient l'homosexualité et la dégénérescence. Dans le contexte

35 Ladame 1893, 534sq.

36 Ladame 1893; Chevalier 1890; Krafft-Ebing 1950; Oosterhuis 1997.

37 Cité par Ladame 1898, 309. 
pénal allemand, ils se positionnent en faveur de la dépénalisation des actes des «invertis-nés», ceux-ci étant porteurs d'une tare, et donc reconnus partiellement responsables de leurs actes. En contre-partie, ils demandent l'extension de la protection de la jeunesse à 18 ou 20 ans. Cette double catégorisation pénale de l'homosexualité est introduite en Suisse dès 1896 dans les avant-projets de CPS, à cause de l'influence de ces deux psychiatres allemands sur Stooss ${ }^{38}$.

Dans les Chronique allemande de 1903 et de 1913, Ladame souligne l'opposition du psychiatre berlinois Magnus Hirschfeld (1868-1935) avec la théorie de la dégénérescence ${ }^{39}$. Les activités d'Hirschfeld sont intimement liées aux mouvements politiques et sociaux de l'époque en Allemagne. En 1897, il crée à Berlin le Wissenschaftlich-humanitäres Komitee (Comité scientifique humanitaire), et lance une pétition pour l'abrogation du $\$ 175$ du code pénal allemand. D'autre part, entre 1899 et 1923, paraît sous sa direction un annuaire consacré à l'homosexualité ${ }^{40}$ où s'expriment les plus grands spécialistes de l'époque, et il sera le cofondateur, avec Forel et le psychiatre anglais Havelock Ellis (1859-1939), de la Ligue mondiale pour la réforme sexuelle en $1921^{41}$.

Reprenant la notion d'uranisme d'Ulrichs, et son idée «d'âme de femme enfermée dans un corps d'homme», Hirschfeld défend l'idée d'un troisième sexe, pour insister à la fois sur le caractère inné de l'orientation sexuelle, et sur les sentiments de pudeur et de moralité propres à tout homme, qu'il soit homosexuel ou normosexuel ${ }^{42}$. En tant qu'expert médical auprès des tribunaux, il obtient souvent l'acquittement d'homosexuels risquant d'être condamnés à la prison en invoquant l'irresponsabilité de leurs actes, car découlant d'un comportement naturel. Sa conception d'une orientation sexuelle naturelle paraît trouver peu d'attention en Suisse. Ainsi, Ladame se montre en totale opposition avec les conceptions théoriques d'Hirschfeld, et dénonce une stratégie de la maladie et de la naturalité pour défendre les homosexuels. De même, Forel, s'il s'accorde avec la théorie du troisième sexe pour expliquer l'homosexualité innée, refuse de la considérer «normale»; la normalité de l'appétit sexuel étant la procréation ${ }^{43}$. Stooss, pour sa part, ne se réfère pas du tout au psychiatre berlinois.

38 Dans son premier projet de 1893, Stooss reprenait les dispositions du droit germanique (Stooss 1893, 262; Stooss 1899, 30; Ladame 1914, 279).

39 Ladame 1903, 304-306; Ladame 1913, 838-840.

40 Le Jahrbuch für sexuelle Zwischenstufen mit besonderer Berücksichtigung der Homosexualität.

41 Ladame 1913, 838-840; cf. Cardon 1993; Tamagne 2000; Steakley 1997.

42 Hirschfeld 1908.

43 Ladame 1913, 841; Forel 1906, 268. 
En outre, la pétition d'Hirschfeld pour l'abolition du $\$ 175$ connaît un sort qui décrédibilise en partie son auteur en Suisse avant la Première Guerre mondiale. En 1905, la pétition portant la signature de quelque 2000 personnalités scientifiques, politiques et artistiques, dont Krafft-Ebing et Moll, provoque un grand débat favorable à la dépénalisation de l'homosexualité au Reichstag. Le débat est soutenu par le député socialiste August Bebel (1840-1913), et la pétition est envoyée en commission. En 1907, une série de scandales et de procès éclaboussent des aristocrates proches du Kaiser, Kuno von Moltke (1848-1916), Philipp von Eulenburg (1847-1921) et Bernhard von Bülow (1849-1929). Appelé comme expert médical dans un des procès de von Moltke, Hirschfeld invoque l'irresponsabilité de «l'uranistené», comme il le fait dans les procès ordinaires. Le parti socialiste allemand, qui s'est montré jusqu'alors le plus fort soutien du Comité scientifique humanitaire, profite de monter une campagne homophobe visant la politique de Guillaume $\mathrm{II}^{44}$. En conséquence des scandales, le rapport de la commission des pétitions du Reichstag se prononce pour le rejet de la pétition, le 23 janvier 1908. Une nouvelle commission chargée de rédiger un avantprojet d'article renforçant les pénalités rend son rapport en 1909. Ce projet introduit la pénalisation de l'homosexualité féminine et de l'abus de détresse spécifiquement homosexuel ${ }^{45}$. Ces deux catégories ne sont finalement pas adoptées par le Reichstag en 1914, mais sont introduites dans l'article pénal suisse, en référence explicite aux motifs allemands ${ }^{46}$, ce qui atteste encore l'influence des théories et des contextes sociopolitiques de l'Allemagne sur la perception médico-légale de l'homosexualité dans la Suisse d'avantguerre.

\section{Les conceptions d'Auguste Forel}

Forel se positionne à plusieurs reprises en faveur de la dépénalisation de l'homosexualité et fait figure de libérateur des homosexuels en Suisse ${ }^{47}$. Se référant aux théories psychiatriques allemandes, Forel traite l'homosexualité de phénomène pathologique, et marque un accord dominant avec les positions de Krafft-Ebing et Moll. Dans la Morale sexuelle, il pose que la plupart des «perversions sont héréditaires et par conséquent innées» ${ }^{48}$. Illustrant la

44 Tamagne 2000; Cardon 1993; Steakley 1989.

45 Ladame 1913.

46 PV CPS III 1913, 177-184.

47 Un psychiatre à la retraite m'a témoigné avoir appris la maxime «Auguste Forel a libéré les homosexuels en Suisse» au cours de ses études.

48 Forel 1907, 30. 
prépondérance du modèle biologique, Forel pose une prédisposition héréditaire à l'homosexualité, comme aux autres anomalies sexuelles, causée par la «blastophthorie» des embryons:

J'entends par blastophthorie, ou détérioration du germe, ce qu'on pourrait appeler aussi la fausse hérédité, c'est-à-dire les suites de toute action directe pathogénique ou perturbatrice, en particulier de certaines intoxications sur les cellules germinatives dont les déterminantes héréditaires sont ainsi changées. La blastophthorie agit donc [sur] les germes non encore conjugués, par l'intermédiaire de leurs porteurs, et crée ainsi, à leur origine, ce qu'on appelle les tares héréditaires de toute nature [...]. L'exemple le plus fréquent de blastophthorie est fourni par l'intoxication alcoolique. ${ }^{49}$

L'homosexualité acquise est, par contre, une perversion due à l'habitude, soit par manque de contact avec le sexe opposé (couvents, prisons, internats), soit par recherche de «situations sexuelles exceptionnelles» ${ }^{50}$. Cependant, Forel estime que la femme peut acquérir plus facilement l'homosexualité que l'homme, car elle distingue moins bien l'amour et les «sensations localisées de volupté», et peut se faire mener à «l'onanisme mutuel» et à une «exaltation psychique» sans l'identifier comme un «sentiment sympathie» ${ }^{51}$. En outre, l'aliéniste lie l'homosexualité féminine à la prostitution en se basant sur son observation des divisions vénériennes des hôpitaux, et la voit comme une «compensation d'un fort appétit sexuel $»^{52}$. La lesbienne est donc vue, soit comme une concurrente de l'homme, soit comme une femme détournée du même homme.

Une fois le type d'homosexualité identifié, Forel propose de la traiter par l'hypnose et par la suggestion de «bonnes habitudes en sens inverse» ${ }^{53}$. Si les potentialités de guérison de l'homosexualité acquise par l'hypnose semblent attestées, il adopte une attitude plus nuancée à l'égard de l'homosexualité innée, «[...] ne trouvant pas une guérison, même bien accentuée, suffisante pour garantir un succès définitif dans des cas de maladies dont les racines sont aussi profondément constitutionnelles ${ }^{54}$.

Contrastant avec ses confrères allemands, Forel mentionne peu d'études de cas d'homosexuel-les. Toutefois, dans les appendices de la Morale sexuelle, il retranscrit la lettre d'un ancien élève d'un collège ecclésiastique ayant développé une attirance homosexuelle, et lui demandant «si par raison de santé et de sentiments, il doit céder à ses appétits homosexuels» ou se marier. Dans sa réponse, l'aliéniste considère:

49 Forel 1906, 35sq., souligné par l'auteur.

50 Forel 1906, 300.

51 Forel 1906, 277sq.

52 Forel 1906, 344.

53 Forel 1906, 491.

54 Forel 1906, 492. 
C'est là une question fort épineuse. Tant que les lois interdisent les rapports homosexuels et en font un crime, il n'est guère possible à un médecin de les permettre, même en cas d'affection nerveuse. Il y a des cas où l'on finit par en arriver à conseiller à de pareils malades d'émigrer dans des pays où l'amour homosexuel n'est pas interdit. Il va sans dire qu'ici j'ai interdit le mariage. .5

Le propos révèle que la limite thérapeutique est légale, et le conseil médical se limite, somme toute, à l'interdiction du mariage pour prévenir la propagation d'une tare héréditaire. Cette interdiction reprend en partie la position de Moll, mais est beaucoup plus novatrice qu'il n'apparaît. Ainsi, Forel écrit dans l'édition de 1906 de La Question sexuelle que le mariage homosexuel «serait fort innocent au point de vue social, et les pauvres diables seraient tranquilles et contents, tout en cessant d'être une menace pour les individus normaux ${ }^{56}$. Cette philanthropie médicale est modifiée dès l'édition suivante, et pose désormais que le code ne doit pas régler ces «mariages pathologiques» ${ }^{57}$.

Forel lie clairement la dépénalisation des actes entre adultes consentant-es avec des mesures de prévention de la propagation de l'homosexualité auprès de la jeunesse et des adultes infirmes. Deux institutions non mixtes sont vues comme des vecteurs de «l'inversion sexuelle», les asiles et les écoles. C. Bonello souligne avec justesse que Forel a surtout observé l'homosexualité dans le cadre de l'institution asilaire ${ }^{58}$. Cependant, son regard ne se limite pas aux divisions des malades mentaux, et porte également sur les internats du personnel. Relatant son expérience sur ce sujet, l'ancien directeur de l'Asile du Burghölzli écrit dans ses Mémoires:

\begin{abstract}
J'eus, à cette époque, l'occasion de voir de plus près une autre catégorie d'individus anormaux, les homosexuels. L'un d'entre eux s'introduisit chez nous en qualité de portier. Il était capable, dévoué, mais il amena à l'asile tout un groupe «d'amis» qui, en cachette, soit entre eux, soit même avec des malades, donnèrent libre cours à leurs anomalies. Je finis par découvrir le pot aux roses. L'un d'entre eux, s'étant amouraché d'un infirmier, avait brodé son nom sur de longues bandes d'étoffe. Ce n'est qu'après que j'eusse éliminé de l'asile tous ces éléments pathologiques, certes dignes de pitié mais dont la présence au sein du personnel était inadmissible, que tout rentra dans l'ordre..$^{59}$
\end{abstract}

La thématique des infirmiers/ères homosexuel-les se retrouve également dans ses écrits sur la non-mixité des institutions publiques, sur les abus sexuels commis sur des malades, et sur le choix de carrières spécifiques avec celles de prêtre catholique et d'instituteur ${ }^{60}$. Dans la même logique, Forel enjoint

55 Forel 1907, 44.

56 Forel 1906, 492sq.

57 Forel 1911, 429; Forel 1922, 433.

58 Bonello 2000, 67.

59 Forel 1941, 135.

60 Forel 1906, 266, 271, 374sq. 
les pédagogues à isoler rapidement les «invertis mineurs» des internats scolaires pour éviter la propagation de l'homosexualité. D'une manière générale, la liberté des homosexuel-les est assortie d'une forte injonction d'exclusion et d'invisibilisation:

Une fois majeurs, qu'ils bâtissent des villes à leur usage, échangent des anneaux de fiançailles et se marient entre individus du même sexe comme ils aimeraient tant le faire; ce serait à notre avis fort innocent, et on devrait les laisser faire. Les adultes normaux peuvent, en somme, fort bien se protéger eux-mêmes de leurs assiduités, dès qu'ils sont prévenus par une instruction suffisante sur les questions sexuelles. L'enfant a droit, au contraire, à être protégé contre toute perversion par contamination, ainsi que contre tout attentat sexuel, de quelque nature qu'il soit, et la société a le devoir d'organiser sa protection. ${ }^{61}$

Pour l'aliéniste, toute sexualité, normale ou déviante, s'insère dans un «socialisme intégral ${ }^{62}$, à la fois élitaire, communautariste, et planificateur sur le plan de la natalité. Ainsi, l'acte homosexuel reste innocent tant qu'il demeure stérile, et est intégré dans un dispositif plus large de «sélection rationnelle» de la race:

Il faut enseigner, ou même imposer, d'une façon exacte le néo-malthusianisme aux malades, aux incapables, aux imbéciles, aux méchants et aux races inférieures. Il faut au contraire tout faire pour pousser les forts, les bien portants, les bons et les esprits supérieurs à se reproduire aussi puissamment qu'ils le peuvent. [...] Un homme capable, actif et bon, donne bien plus à la société qu'il ne lui prend, et constitue par là une valeur économique. Un invalide de corps et d'esprit, de même qu'un mauvais sujet, prend au contraire à l'ensemble plus qu'il ne lui donne, et constitue ainsi non seulement une non-valeur, mais un déficit économique. ${ }^{63}$

La dépénalisation de l'homosexualité prend son sens dans des conceptions eugénistes promouvant l'acte sexuel sans procréation pour certaines classes d'individus. En définissant la forme d'homosexualité tolérée, en enjoignant aux homosexuel-les de s'auto-exclure, et en appelant à une prophylaxie de l'homosexualité, les écrits de Forel illustrent la mise en place d'un dispositif plus vaste de contrôle de la sexualité. J. Katz souligne qu'au début du $\mathrm{XX}^{\mathrm{e}}$ siècle, le terme d'hétérosexualité, dans le sens d'acte sexuel entre un homme et une femme sans procréation, tend à signifier une déviance ${ }^{64}$. On peut voir d'une manière plus générale que la construction de la définition pénale de la «débauche contre nature», la perte de la semence dans un sens laïcisé, la cantonne à l'homosexualité, et laisse libre court à la diffusion d'idées légitimant l'hétérosexualisation du plaisir de la part d'une nouvelle

61 Forel 1906, 542.

62 Forel 1912. En outre, Forel adhère au Parti socialiste suisse en 1916 (Forel 1941).

63 Forel 1906, 517sq., souligné par l'auteur. Par exemple, Forel mentionne dans toutes les éditions françaises et allemandes de La Question sexuelle la même adresse à Paris pour commander des condoms réutilisables et encourage les médecins à la diffusion de son utilisation.

64 Katz 2001. Sur l'effet d'imposition des théories médicales, cf. Greenberg 1998; Rosario 2000; Foucault 1976. 
élite, qui s'affirme dans les cas de Ladame et de Forel en rupture avec les codes de l'Eglise, les psychiatres ${ }^{65}$.

\section{Sous le signe de la dégénérescence}

En Suisse, la médicalisation de l'homosexualité est profondément basée sur la notion de dégénérescence au début du $\mathrm{XX}^{\mathrm{e}}$ siècle. Ainsi, les conceptions médico-légales de l'homosexualité s'accordent nettement avec Krafft-Ebing et Moll qui la considèrent comme une anormalité à encadrer. A l'inverse, la théorie de la naturalité défendue par Hirschfeld est contestée, et son action en faveur d'une totale dépénalisation de l'homosexualité se voit décriée suite aux scandales de 1907, notamment par Ladame ${ }^{66}$.

Avant la Première Guerre mondiale, la théorie de la dégénérescence explique la cause de l'homosexualité innée, et, dans le même temps, l'homosexualité devient une preuve de la «dégénérescence», une perversion dont il faut protéger la société. Ce glissement entre biologisme et prédiction sociale est particulièrement clair chez Ladame, et à mettre en parallèle avec Forel: l'homosexualité est provoquée «par l'amoindrissement fonctionnel des centres nerveux inférieurs» et par «la domestication (suite de l'éducation civilisée) qui paralyse la volonté» pour le penchant «naturel» vers l'autre sexe ${ }^{67}$. L'homosexualité éclaire par la marge la construction de la dégénérescence comme principe qui, agité face à tous les désordres dans la psychiatrie et la société suisses, renforce les discours réactionnaires sur toutes sortes de classes d'individus ${ }^{68}$.

65 La rupture avec l'interdit chrétien n'est pas totale si l'on juge l'«écœurement» maintes fois écrit par Ladame et Forel pour qualifier l'homosexualité. D'autre part, la grande majorité des psychiatres suisses mentionnés dans cet article est d'origine protestante, et une analyse plus fine sur les effets de cette socialisation reste à faire. Pour une lecture contemporaine du rapport entre l'Eglise réformée suisse et l'homosexualité, cf. Graesslé/Bühler/Müller 2001.

66 Les références à Hirschfeld par les médecins et les juristes suisses sont moins fréquentes que celles à Krafft-Ebing et Moll avant la Première Guerre mondiale. Hirschfeld gagnera une audience plus large en Suisse au cours des années 1920, mais y restera toujours suspect en raison de sa «propagande» en faveur de l'homosexualité.

67 Ladame 1913, 854-856, souligné par l'auteur. On peut relever dans ces propos une continuité avec les liens opérés entre la dégénérescence et l'homosexualité depuis la moitié du $\mathrm{XIX}^{\mathrm{e}}$ siècle en France, et dont les effets sont encore à analyser. Sur les théories françaises, cf. Aron/Kempf 1978; Rosario 2000; Mendès-Leite 2000.

68 Pour une étude sur le concept de dégénérescence en Suisse, cf. Jeanmonod 1998. Cf. également Jost 1992. 


\section{Bibliographie}

\section{Abréviations utilisées}

FF IV 1918 = «Message du Conseil Fédéral à l'Assemblée fédérale à l'appui d'un projet de code pénal suisse (du 23 juillet 1918)», Feuille Fédérale IV (1918) 1 et s.

FF II 1985 = «Message concernant la modification du code pénal et du code pénal militaire (Infractions contre la vie et l'intégrité corporelle, les mœurs et la famille)», Feuille Fédérale II (1985) 1021 et s.

PV CPS III 1913 = Code pénal suisse. Procès-verbal de la deuxième commission d'experts, vol. III avril 1913 (1914 éd.)

SSP, $1910 / 2=« 43^{\mathrm{e}}$ protocole de la réunion de la SMAS des 26 et 27 novembre 1910», Archives de la Société Suisse de Psychiatrie (1910 non publié)

SSP, $1911 / 1=$ « $44^{\mathrm{e}}$ protocole de la réunion de la SMAS des 5 et 6 juin 1911», Archives de la Société Suisse de Psychiatrie (1911 non publié)

SSP, $1914=\ll 50^{\mathrm{e}}$ protocole de la réunion du printemps de la SMAS des $1^{\text {er }}$ et 2 avril 1914», Archives de la Société Suisse de Psychiatrie (1914 non publié)

Traverse 2003/1 = «Psychiatriegeschichte in der Schweiz (1850-2000). L'histoire de la psychiatrie en Suisse (1850-2000)», Traverse. Zeitschrift für Geschichte. Revue d'histoire 1 (Zurich 2003)

\section{Documents sources}

Bleuler, Eugen, Lehrbuch der Psychiatrie (Berlin 1916)

Chevalier, Julien, «De l'inversion sexuelle aux points de vue clinique, anthropologique et médico-légal», Archives d'anthropologie criminelle et de médecine légale 5 (1890) 314-336

Forel, Auguste, La question sexuelle exposée aux adultes cultivés (Paris ${ }^{21906)}$

- La Morale sexuelle (Lausanne 1907)

- La question sexuelle exposée aux adultes cultivés (Paris ${ }^{31911)}$

- Pensée, Liberté et Socialisme. Buts de la Libre Pensée (Lausanne 1912)

- La question sexuelle (Paris $\left.{ }^{5} 1922\right)$

- Mémoires (Neuchâtel 1941)

Hafter, Ernst, «Homosexualität und Strafgesetzgeber», Revue pénale suisse 43 (1929) 37-71

Hirschfeld, Magnus, Les homosexuels de Berlin, 1908 (1908, rééd. Lille 2001)

Krafft-Ebing, Richard von, Psychopathia sexualis. Etude médico-légale à l'usage des médecins et des juristes. Edition refondue par le Dr Albert Moll (Paris 1950, rééd. 1999)

Ladame, Paul-Louis, "Chronique allemande», Archives d'anthropologie criminelle et de médecine légale 8 (1893) 526 et s.

- «Chronique allemande», Archives d'anthropologie criminelle et de médecine légale 13 (1898) 297-312

- «Chronique allemande», Archives d'anthropologie criminelle et de médecine légale 18 (1903) 292-314

- «Chronique allemande», Archives d'anthropologie criminelle et de médecine légale 28 (1913) $827-861$

- «L'homosexualité dans l'Avant-projet du Code pénal suisse. Remarques psychiatriques sur l'article du nouvel Avant-projet de 1913», Revue pénale suisse (1914) 279-295

Picot, Ernest, «Les délits contre les mœurs dans les codes pénaux suisses. Etude de législation comparée», Revue pénale suisse (1889) 51-67

Praetorius, Numa, «A propos de l'homosexualité en Allemagne», Archives d'anthropologie criminelle et de médecine légale 27 (1912) 114-116

Repond, André, «Les tendances actuelles de la psychiatrie en Suisse», extrait des Annales Médico-Psychologiques 5 (Paris 1936)

Stooss, Carl, Die Grundzüge des Schweizerischen Strafrechts (Basel/Genf 1893)

- Bericht über den Vorentwurf zu einem Schweizerischen Strafgesetzbuch nach den Beschlüssen der Expertenkommission (Bern 1899) 


\section{Documents contemporains}

Aron, Jean-Paul/Roger Kempf, Le Pénis et la Démoralisation de l'Occident (Paris 1978)

Bomio, Giorgio, «Forel et le droit pénal - Influence d'un psychiatre sur la préparation du code pénal suisse», Revue pénale suisse 107 (1990) 87-105

Bonello, Christian, «Du médecin légiste à l'aliéniste: l'homosexualité sous le regard de la médecine au XIX ${ }^{\mathrm{e}}$ siècle», dans: Louis-Georges Tin/Geneviève Pastre, Homosexualités: expression/répression (Paris 2000) 65-81

Cardon, Patrick, «Préface à Dr Magnus Hirschfeld», dans: Magnus Hirschfeld, Les Homosexuels de Berlin, 1908 (Lille 1993)

Feray, Jean-Claude/M. Herzer, «Kertbeny, une énigmatique 〈mosaïque d'incongruité»», Etudes finno-ougriennes XXII (1990) 215-239

Foucault, Michel, Histoire de la sexualité. La volonté de savoir (Paris 1976)

Fussinger, Catherine/Deodaat Tevaearai, Lieux de folie, monuments de raison (Lausanne 1998)

Gasser, Jacques/Vincent Barras, «Les psychiatres et le code pénal: débats à la Société des médecins aliénistes suisses au tournant du siècle», Archives suisses de neurologie et de psychiatrie 151 Suppl (2000) 15-19

- «La responsabilité limitée, histoires et problèmes», dans: Patients psychiatriques en prison (Lausanne 2000a) 47-53

- «Essai de bibliographie sur l'histoire de la psychiatrie en Suisse», Archives suisses de neurologie et de psychiatrie 151 Suppl (2000b) 44-62

Gerodetti, Natalia, Modernising Sexualities. Towards a Socio-Historical Understanding of Sexualities in the Swiss Nation (Bern 2005)

Graesslé, Isabelle/Pierre Bühler/Christoph D. Müller, Qui a peur des homosexuel-les? (Genève 2001)

Greenberg, David F., The Construction of Homosexuality (Chicago 1998)

Jeanmonod, Gilles, «La mutation du concept de dégénérescence en Suisse romande 1870-1920» Gesnerus 55 (1998) 70-86

Jost, Hans Ulrich, Les avant-gardes réactionnaires. La naissance de la nouvelle droite en Suisse. 1890-1914 (Lausanne 1992)

Katz, Jonathan Ned, L'invention de l'hétérosexualité (Paris 2001)

Kennedy, Hubert, «Karl Heinrich Ulrichs, First Theorist of Homosexuality», in: Vernon A. Rosario (editor), Science and Homosexualities (New York/London 1997) 26-45

- The Ideal Gay Man. The Story of Der Kreis (New York/London 1999)

Leroy-Forgeot, Flora, Histoire juridique de l'homosexualité en Europe (Paris 1997)

Mendès-Leite, Rommel, Le sens de l'altérité. Penser les (homo)sexualités (Paris 2000)

Oosterhuis, Harry, "Richard von Krafft-Ebing's 'Step-Children of Nature'. Psychiatry and the Making of Homosexual Identity", in: Vernon A. Rosario (editor), Science and Homosexualities (New York/London 1997) 67-88

Rosario, Vernon A., L'irrésistible ascension du pervers. Entre littérature et psychiatrie (Paris 2000)

Steakley, James D., "Iconography of a Scandal: Political Cartoons and the Eulenburg Affair in Wilhelmin Germany", in: Martin Duberman/Martha Vicinius/George Chauncey, Hidden from History. Reclaiming with the Gay and Lesbian Past (New York 1989)

- "Per scientiam ad justitiam. Magnus Hirschfeld and the Sexual Politics of Innate Homosexuality", in: Vernon A. Rosario (editor), Science and Homosexualities (New York/London 1997) 133-154

Tamagne, Florence, Histoire de l'homosexualité en Europe - Berlin, Londres, Paris, 1919-1939 (Paris 2000)

Trüeb, Kuno/Stephan Miescher, Männergeschichten. Schwule in Basel seit 1930 (Basel 1988) 